Review

\title{
Research Advances in CKLF-like MARVEL Transmembrane Domain-containing Family in Non-small Cell Lung Cancer
}

\author{
Keheng $\mathrm{Wu}^{1 \#}$, Xiaoman $\mathrm{Li}^{2 \#}$, Huadi Gu${ }^{3}$, Qiao Yang1, Yingying Liu1 ${ }^{1}$, and Liang Wang ${ }^{3 凶}$ \\ 1. China Medical University-The Queen's University of Belfast Joint College, Shenyang, Liaoning, 110122, China \\ 2. Institute of Translational Medicine, Key Laboratory of Medical Cell Biology, Ministry of Education, China Medical University, Shenyang, Liaoning, 110122, \\ China \\ 3. Department of Pathology, College of Basic Medical Sciences, China Medical University, Liaoning, 110122, China \\ \#: These authors contributed equally to this study. \\ $\triangle$ Corresponding author: Liang Wang, MD, PhD., Department of Pathology, College of Basic Medical Sciences and the First Affiliated Hospital, China Medical \\ University, Shenyang, 110001, China. E-mail address: cmuwangliang@qq.com
}

(C) The author(s). This is an open access article distributed under the terms of the Creative Commons Attribution License (https://creativecommons.org/licenses/by/4.0/). See http:/ /ivyspring.com/terms for full terms and conditions.

Received: 2019.02.01; Accepted: 2019.08.12; Published: 2019.09.07

\begin{abstract}
CKLF-like MARVEL transmembrane domain-containing member (CMTM) is a new gene family first cloned and reported in 2001. The CMTM family consists of nine members including CKLF and CMTM1-CMTM8, which are located on different chromosomes. Besides exhibiting extensive chemotactic activity, the CMTM family plays an important role in the hematopoiesis system, the immune system, the cardiovascular system and the male reproductive system. Recent in-depth research has also revealed that CMTM is closely associated with the genesis, development and metastasis of tumors, displaying opposing activities in diverse human tumors. In this review, we discuss the structural and functional characteristics of the CMTM family and summarize latest research findings of the relationship between several CMTM members and non-small cell lung cancer.
\end{abstract}

Key words: CMTM; CKLFSF; MARVEL; tumor suppressor gene; non-small cell lung cancer

\section{Introduction}

Chemokine-like factor superfamily members (CKLFSF) are part of a novel gene family that was first described in 2001 [1, 2]. Han et al. cloned a novel cytokine, chemokine-like factor 1 (CKLF1), from the phytohemagglutinin-stimulated leukemia cell line U937, which was used to study interleukin-10 inhibition by suppression subtractive hybridization. They also cloned and validated CKLF-like MARVEL transmembrane domain-containing members (CMTM) by reverse transcription PCR [2-4]. In 2005, the International Human Genetics Nomenclature Committee renamed CKLFSF1-8 as CMTM1-8 according to their molecular structures [5-7]. CMTM1-8 and chemokine-like factor $(C K L F)$, are located on different chromosomes. CKLF and CMTM1-4 are grouped together on chromosome 16q22.1 to form a gene cluster, CMTM5 is independently located on 14q11.2, and CMTM6-8 are co-located on chromosome 3p23. Their coding products include chemokines and the transmembrane 4 superfamily (TM4SF). CMTM1 in particular shows the most similarity to chemokines, CMTM8 resembles TM4SF, and the biological features of CMTM2-7 are somewhere in between [2, 8-15].

Functionally, CMTMs not only have broad-spectrum chemotactic activity, but also play an important biological role in the hematopoietic, immune, cardiovascular, and male reproductive systems [16-20]. Additionally, CMTMs are associated with autoimmune and hematopoietic diseases [21-23], and with the genesis, development, and metastasis of multiple malignancies [24-27]. Each CMTM member 
has different biological functions in different tumors, although they collectively play a suppressive role. Many cancers of the digestive tract such as esophageal, gastric, and oral cancer involve CMTM3 in several different pathways [28-33], while, cancers of digestive glands, like hepatocellular carcinoma, are also associated with CMTM members [34, 35]. Moreover, researches in genitourinary cancers indicate that CMTM3 and CMTM8 suppress tumor cell reproduction and migration [36-40].

Isoform CMTM1_v17 of CMTM1, a newly discovered member of the family, functions in breast cancer carcinogenesis and is a potential novel therapeutic target [41]. CMTM1 overexpression in the glioblastoma cell line A172 also promotes the proliferation and migration of tumor cells, which is likely to be achieved by the activation of epidermal growth factor receptor (EGFR), Src family kinase, and Wnt signaling [42]. However, the specific mechanism of CMTM1 in the development of glioblastoma is unclear. Moreover, although CMTM3 showed anti-cancer effects in testicular, prostate, liver, gastric, and kidney cancers as well as other tumors, it had a cancer-promoting effect in glioma [42]. Similarly, CMTM7 showed a cancer-promoting role in glioma but an anti-cancer role in non-small cell lung cancer (NSCLC) [42, 43], while CMTM4, CMTM5, and CMTM8 functioned in tumor suppression in a variety of solid tumors [44-46]. Thus, the functions of CMTM family members are diverse in different malignancies, so it is important to further study their roles and mechanisms in tumorigenesis, development, and metastasis. This could identify new molecular targets for tumor detection and gene therapy, bringing new hope for cancer patients. A review of the literatures suggests that several CMTMs are closely involved in the malignant progression of NSCLC, and we discuss their relationship in the current review, with particular focus on CMTM7.

\section{CMTMI and NSCLC}

CMTM1 is located on chromosome 16 and encodes at least 23 alternative spliced isoforms (CMTM1_v1-v23) [47]. The CMTM1_v1-16 protein is encoded by open reading frame (ORF)1 while ORF2 encodes CMTM1_v17-23 [41]. CMTM1_v17 consists of 149 amino acids and has clear tissue expression specificity, being highly expressed in the testes and prostate tissues, but low or undetectable in many other tissues [48]. Recent studies have shown that CMTM1_v17 is also highly expressed in a variety of solid tumors (breast cancer, kidney cancer, lung cancer, and ovarian cancer) and that it can activate nuclear factor-kappa B (NF-kB) signaling to promote cell proliferation and partially resist apoptosis induced by tumor necrosis factor-a [41]. Additionally, CMTM1_v17 expression is strongly linked with chemotherapy resistance and poor prognosis in early stage NSCLC patients who have received neoadjuvant chemotherapy [49]. Protein Atlas search found that lower CMTM1 expression was associated with a higher survival probability in both lung squamous cell carcinoma and adenocarcinoma (Figure 1).

\section{CMTM5 and NSCLC}

CMTM5 is located at $14 \mathrm{q} 11.2$ and does not cluster with any other CMTM members, but is closely linked to the interleukin 25 gene [50]. It includes at least six mRNA splicing bodies, CMTM5-v1-v6, of which CMTM5-v1 is the most conserved. CMTM5 is widely expressed in many normal tissues such as brain, prostate and small intestine, and has a secretory form [51], although its expression is greatly reduced in many tumor tissues, such as prostate cancer, cervical cancer, ovarian cancer, leukemia, glioma, and
(A)

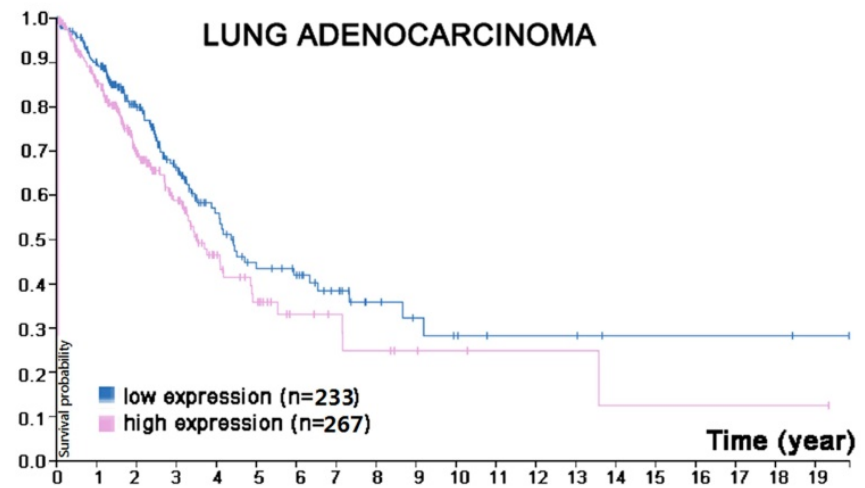

(B)

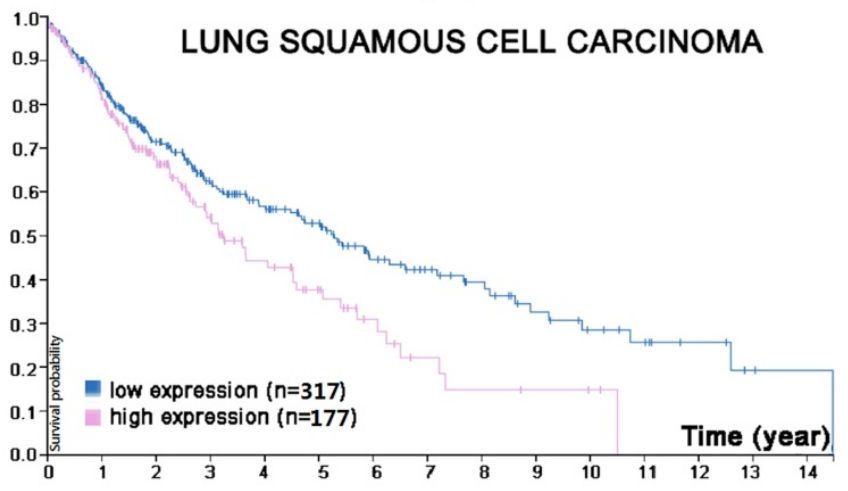

Figure 1. In the course of lung adenocarcinoma (A) and lung squamous cell carcinoma (B) progression, lower CMTM1 expression was associated with a higher survival probability $(p<0.053$ for lung adenocarcinoma, $p<0.013$ for lung squamous cell carcinoma). (Image credit: Human Protein Atlas. https://www.proteinatlas.org/ENSG00000089505-CMTM1/pathology/tissue/lung+cancer) 
digestive system tumors [52-56]. Its anti-cancer mechanism are not clear, but may be related to promoter methylation or the function of multiple signal transduction pathways [11]. A nude mouse model of prostate cancer xenografts was established to confirm the inhibitory effect of CMTM5 [55]. After 3 weeks, CMTM5 adenovirus was injected into the tumor, and CMTM5 was shown to inhibit the growth of prostate cancer by downregulating the expression of vascular endothelia growth factor and NF- $\mathrm{kB}$ [51, 55]. Another team confirmed that CMTM5 inhibits the proliferation and migration capacity of prostate cancer cells [54]. Moreover, a Protein Atlas search found that reduced CMTM5 expression was associated with a lower survival probability in lung adenocarcinoma and squamous cell carcinoma (Figure 2).

\section{CMTM6 and NSCLC}

CMTM6 is another member of the CMTM superfamily located at 3 p22.3 adjacent to CMTM7. It is 21600 nucleotides long and encodes three transcripts, CMTM6-001 to CMTM6-003, but only CMTM6-001 with four introns can be successfully be translated. CMTM6 is a 183 amino acid protein with a typical MARVEL domain, and is mainly expressed in the lung, liver, immune organs like the spleen, lymph nodes, and tonsils, as well as reproductive organs [2]. CMTM6 also showed clinical importance in hepatocellular carcinoma (HCC) [57]. Histological revealed higher CMTM6 expression in HCC tissues than in adjacent normal tissues, and its expression correlates with metastasis, tumor staging and the expression of alpha-fetoprotein. High CMTM6 expression is also associated with poor prognosis in malignant gliomas, which is caused by the inhibition of T-cell-mediated anti-tumor immunity [58]. This finding is consistent with the previously discovered

(A)

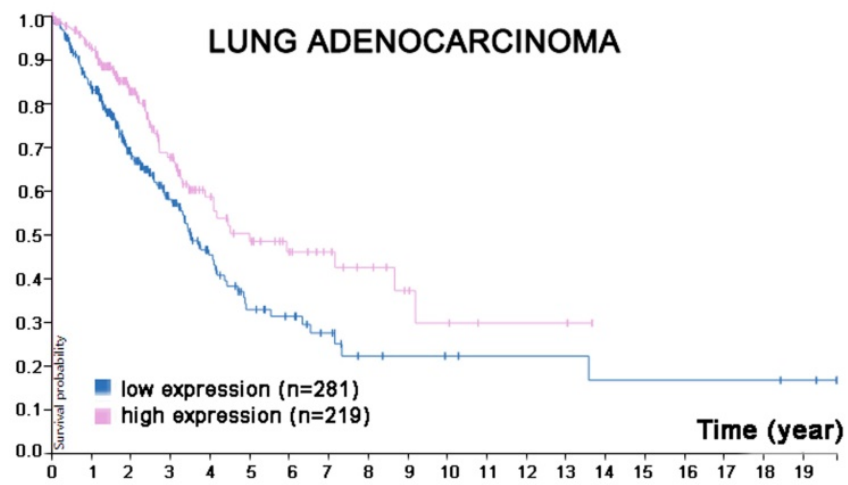

identity of CMTM6 as a key regulator of programmed cell death ligand 1 (PD-L1), which functions as an immune checkpoint inhibitor of $\mathrm{T}$ cells $[26,59]$. Mezzadra et al. [26] and Burr et al. [59] recently reported the regulation of PD-L1 by CMTM6 and its pro-oncogenic role in several cell lines following clustered regularly interspaced palindromic repeats (CRISPR)-CRISPR-associated protein (Cas)9 deletion of CMTM6 and subsequent decrease in PD-L1 expression. CMTM6 was shown to co-localize with PD-L1 on the plasma membrane and recycling endosomes, aiding its return to the cell surface and impeding its degradation. It enhanced the resistance of PD-L1 to endoglycosidase $\mathrm{H}$, preventing it from undergoing deglycosylation.

Mezzadra also transfected the NSCLC cell line H2030 with short hairpin (sh)RNA against CMTM6, either exclusively or in combination with shRNA against CMTM4, and found out that while the absence of CMTM6 decreased PD-L1, CMTM4 could attenuate this effect [26]. Similar results were obtained in another NSCLC cell line, H2122. Moreover, Burr et al. carried out RNA interference and CRISPR-Cas0 knockout of CMTM6 in the lung adenocarcinoma cell line, HCC-827, resulting in the reduction of PD-L1 [59]. A Protein Atlas search of the relationship between CMTM6 mRNA expression and prognosis was consistent with these findings (Figure 3 ).

\section{CMTM7 and NSCLC}

\section{Features of CMTM7 and expressed products}

CMTM7 also belongs to the CMTM superfamily, is located on chromosome 3p22.3 between CMTM6 and CMTM8 and within the same cluster, and is 63858 bp in length. It is highly expressed in leucocytes and has six splicing isoforms: CMTM7-001 to CMTM7-006. Of these, CMTM7-003 is not translated because of its retained introns, while CMTM7-004 and CMTM7-006
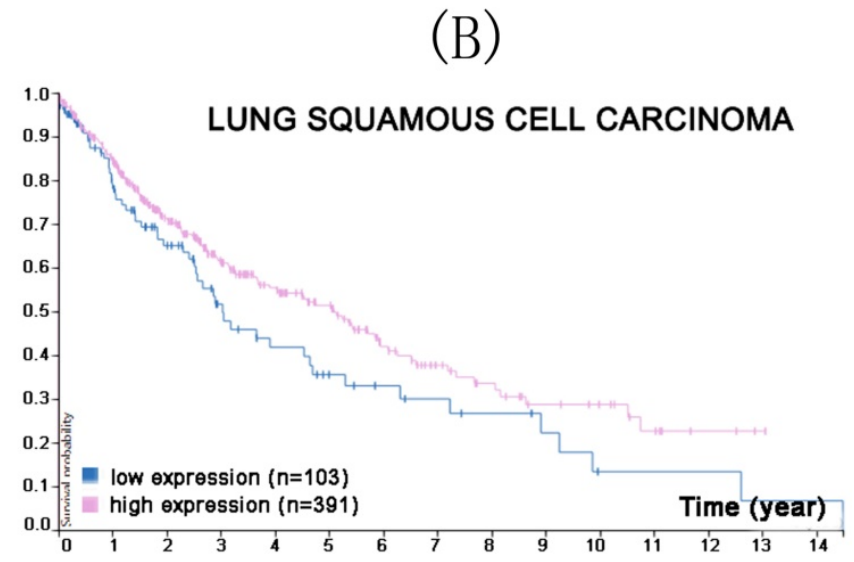

Figure 2. In the course of lung adenocarcinoma (A) and lung squamous cell carcinoma (B) progression, reduced CMTM5 expression was associated with a lower survival probability $(p<0.0021$ for lung adenocarcinoma, $p<0.057$ for lung squamous cell carcinoma). (Image credit: Human Protein Atlas. https://www.proteinatlas.org/ENSG00000166091-CMTM5/pathology/tissue/lung+cancer) 
are not translated because of nonsense-mediated mRNA decay. The main splicing isoform (CMTM7-001) can be detected by northern blotting; its cDNA is 1369 bp long, including four introns, five exons, a classical promoter sequence, and a poly(A) tail [60]. CMTM7 mRNA expression in healthy human tissues shows organ specificity, with significantly higher expression observed in the spleen, breast, and lung (Figure 4).

\section{CMTM7 is associated with the survival of lung cancer patients}

Liu [43] reported that CMTM7 was mainly expressed in the nuclei of tumor tissues of 127 lung adenocarcinoma patients, but in the cytoplasm and cell membrane of adjacent normal tissues. Total expression was either increased (43/127), decreased $(54 / 127)$, or remained unchanged $(30 / 127)$ in these patients, with abnormal expression always associated with a lower survival rate [43]. This indicated an important role for CMTM7 in lung cancer. Figure 5 shows the connection between CMTM7 expression and patient survival probability.

CMTM7 mainly affects the EGFRphosphoinositide 3-kinase (PI3K)/protein kinase B (AKT) pathway by upregulating the expression of p27 and downregulating cyclin-dependent kinase 2 (CDK2) and CDK6 to arrest the cell cycle at G1/S phase and slow cell growth [61]. In cells transfected with CMTM7, more EGFR was transferred from the cell membrane to the cytoplasm and degraded than in control cells, and AKT showed decreased phosphorylation.

To further understand the molecular mechanism of CMTM7 within NSCLC, Liu [62] carried out CMTM7 knockdown in A549 cells. They observed delayed degradation and increased internalization of EGFR, which are closely connected with the transportation and fusion of endosomes. CMTM7 knockdown also decreased EGFR ubiquitination, which further delayed its degradation. Moreover, CMTM7 co-localized with Rab5 and EEA1 in early endosomes and aided endosome fusion. However, it

(A)

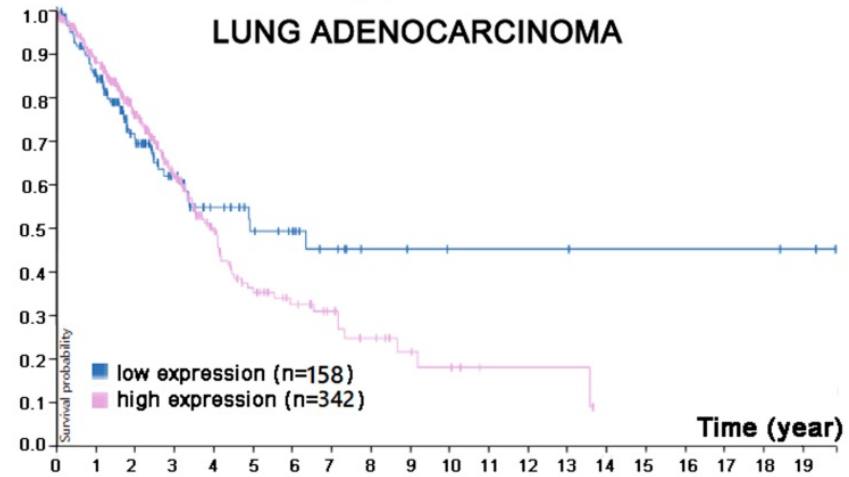

(B)

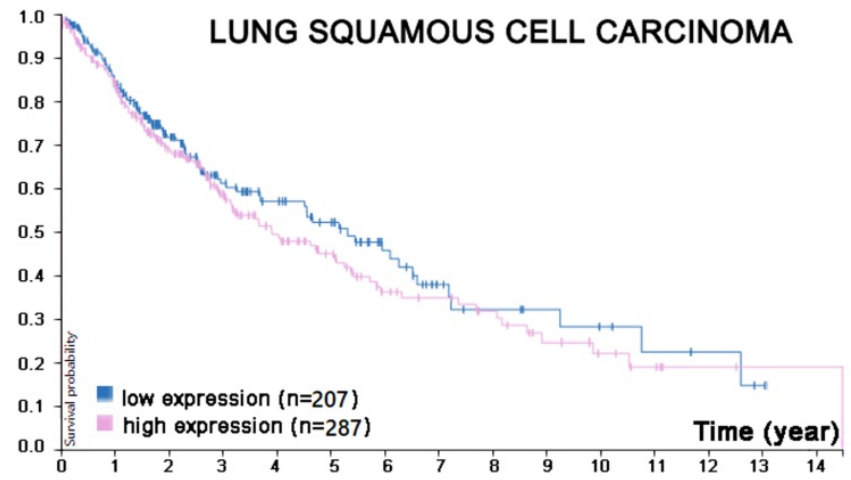

Figure 3. In the course of lung adenocarcinoma (A) and lung squamous cell carcinoma (B) progression, higher CMTM6 expression was associated with a lower survival probability $(p<0.4$ for lung adenocarcinoma, $p<0.33$ for lung squamous cell carcinoma). (Image credit: Human Protein Atlas. https://www.proteinatlas.org/ENSG00000091317-CMTM6/pathology/tissue/lung+cancer)

GTEx dataset RNA tissue category: Expressed in all

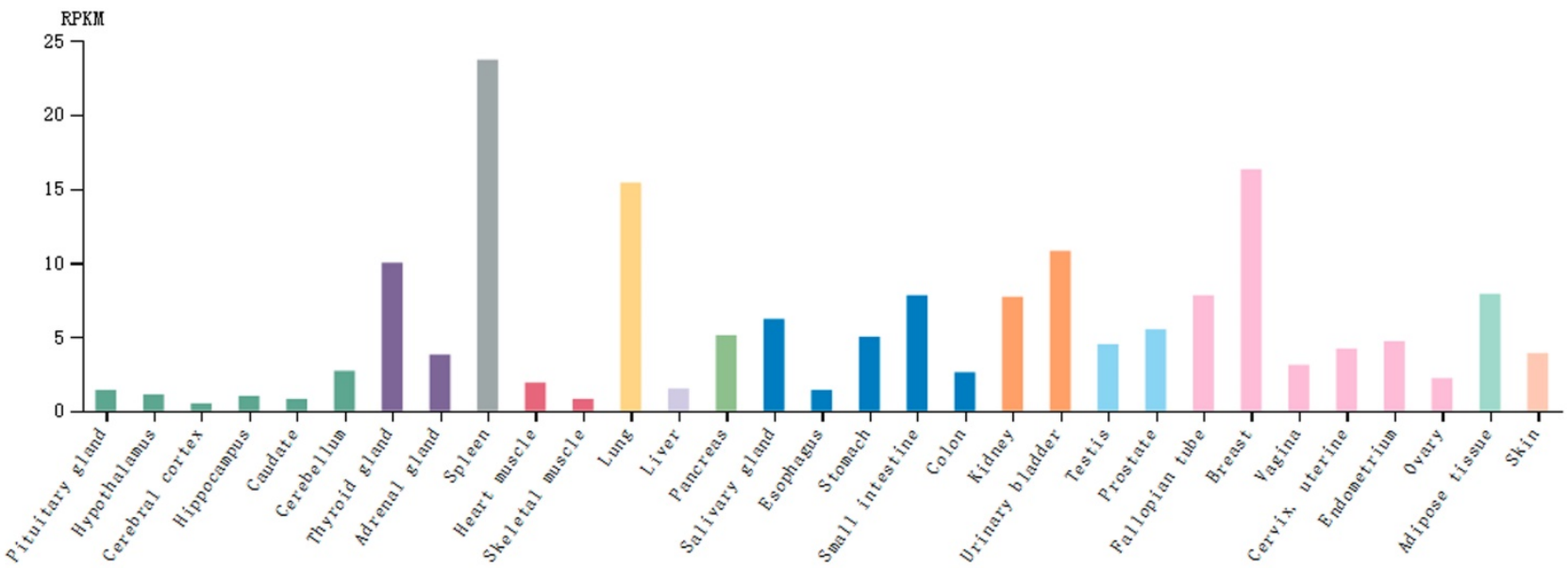

Figure 4. CMTM7 mRNA expression is relatively higher in tissues from immune, hematopoietic, lung, and reproductive organs. (Image credit: Human Protein Atlas) 
inhibited the interaction of Rab5 and Rabaptin5, which is a critical step that affects fusion. That suggested that CMTM7 blocks the EGFR-AKT pathway by promoting EGFR internalization via endosomes (Figure 6).

Additional research by Liu [63] probed the effect of RNA interference in knocking down the expression of CMTM7 in A549 cells. This showed that CMTM7 sustained and promoted the growth of tumor cells and inhibited apoptosis, while flow cytometry detected relatively more cells in G0/G1 phase than in $\mathrm{S}$ or G2/M phase. Together, these findings indicated that CMTM7 inhibits apoptosis in a "tumor promoting" mechanism through influencing the cell cycle. However, the contradiction of this finding with other studies and the unclear mechanism of action reflect the complexity of the relationship between CMTM7 and lung cancer, suggesting a need for further research.

\section{CMTM8 and NSCLC}

CMTM8 is located on chromosome 3p23 and its full length of cDNA is $1185 \mathrm{bp}$, of which nucleotides 295-816 encode CMTM8 [8]. The expression product is a four-time transmembrane protein consisting of 173 amino acids and MARVEL domains for vesicular transport and membrane ligation [8]. CMTM8 is widely expressed in many normal human tissues and is frequently downregulated or absent in multiple solid tumors (liver, lung, colon, rectum, esophagus, and stomach) [13, 36, 37, 64, 65]. CMTM8 inhibition promotes tumor cell proliferation, migration and
(A)

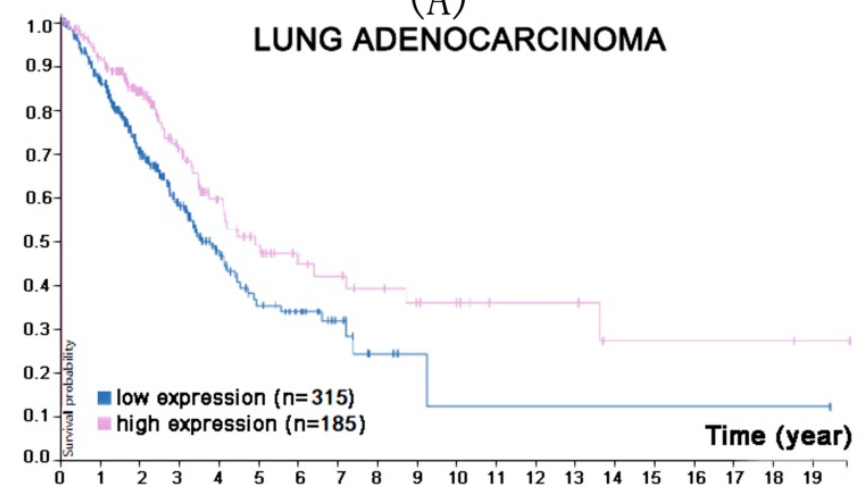

(B)

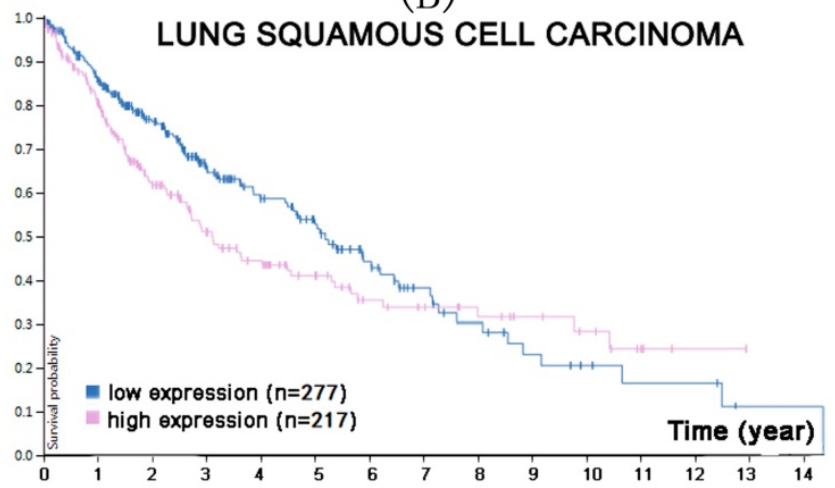

Figure 5. In the course of lung adenocarcinoma (A) and lung squamous cell carcinoma (B) progression, lower CMTM7 expression was associated with a lower survival probability for lung adenocarcinoma $(p<0.0066)$, but a higher survival probability for lung squamous cell carcinoma $(p<0.058)$. (Image credit: Human Protein Atlas. https://www.proteinatlas.org/ENSG00000153551-CMTM7/pathology/tissue/ lung+cancer)

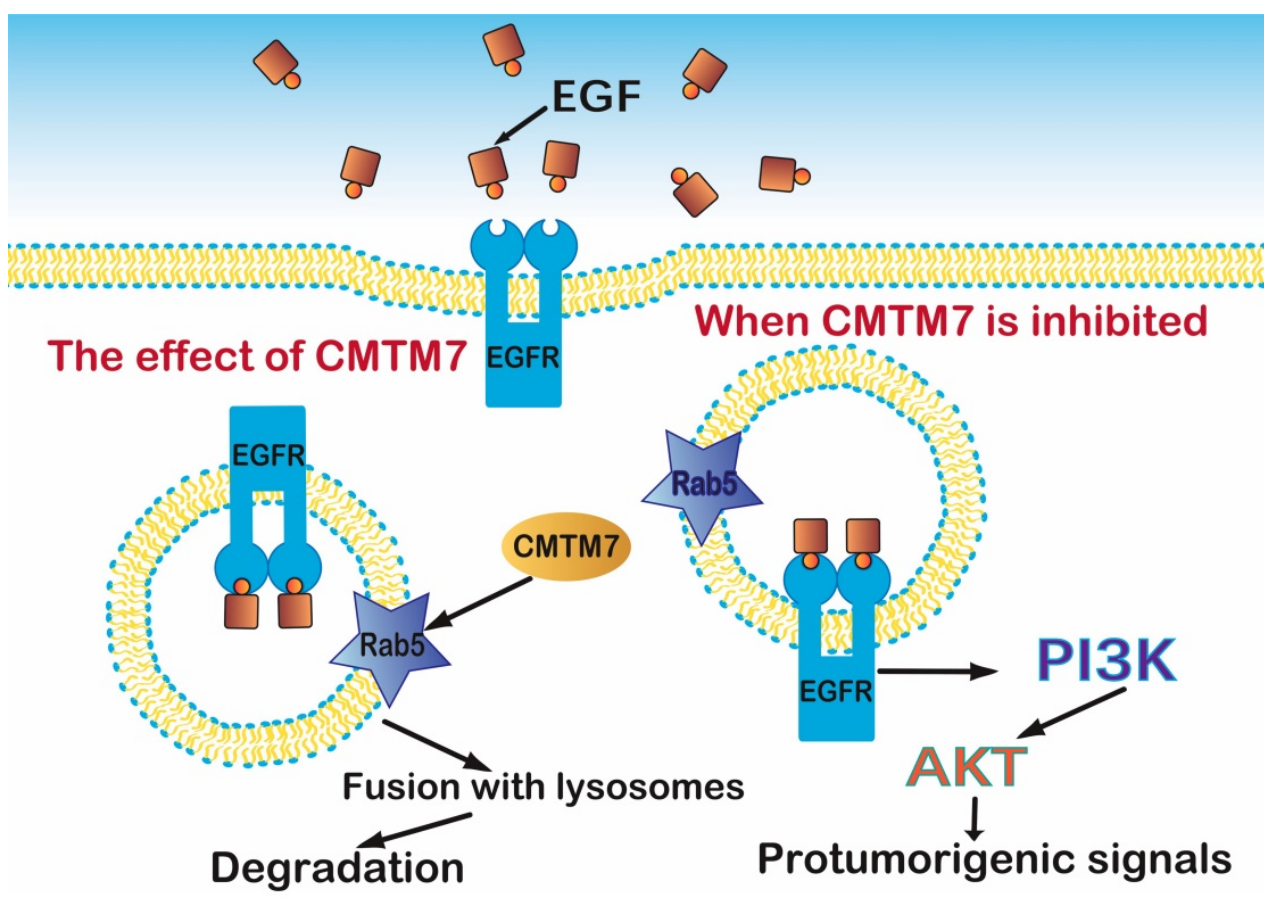

Figure 6. CMTM7 activates Rab5 which controls the fusion of early endosomes and affects EGFR protein trafficking, an essential part of the PI3K/AKT signaling pathway. 
(A)

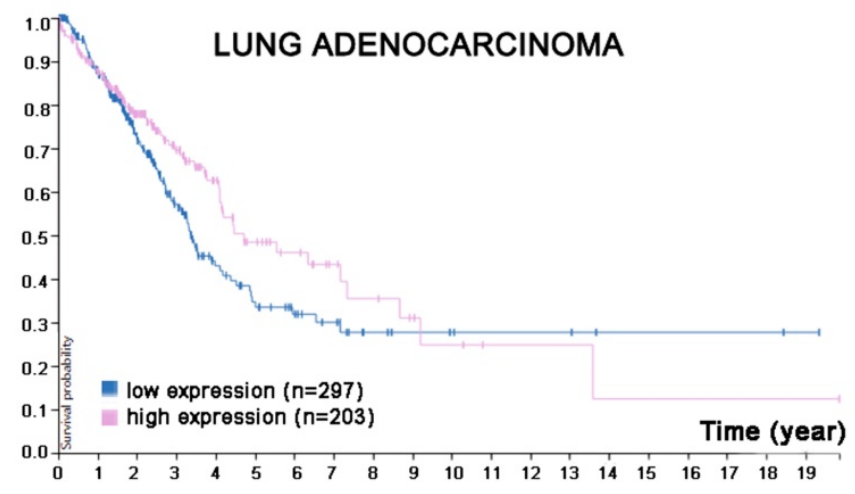

(B)

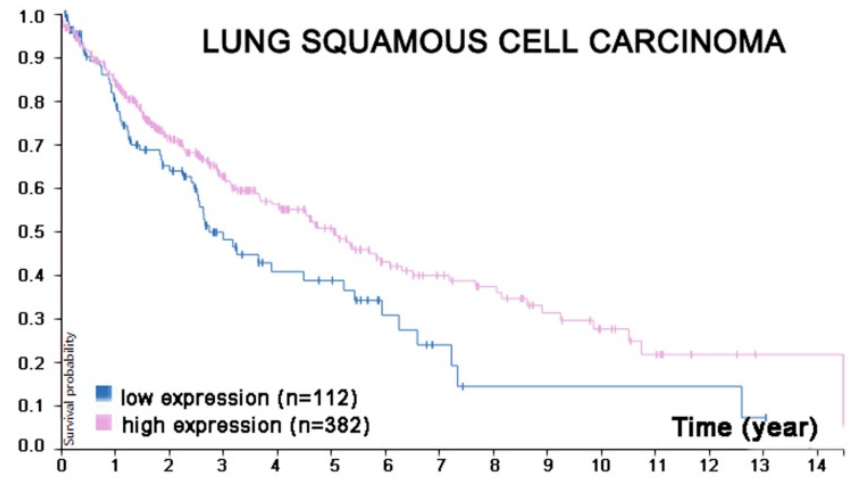

Figure 7. In the course of lung adenocarcinoma (A) and lung squamous cell carcinoma (B) progression, lower CMTM8 expression was associated with a lower survival probability $(p<0.099$ for lung adenocarcinoma, $p<0.018$ for lung squamous cell carcinoma). (Image credit: Human Protein Atlas. https://www.proteinatlas.org/ENSG00000170293-CMTM8/pathology/tissue/lung+cancer)

invasion [8]. Although the anti-tumor mechanism of CMTM8 is unclear, it is thought to accelerate the internalization of transferrin receptor and EGFR, and to be involved in a number of signal pathways associated with tumorigenesis and development [8, $14,15]$. To elucidate further details, it is important to study the relationship between CMTM8 and a diverse range of tumor with the aim of identifying new targets of tumor gene therapy [13]. A Protein Atlas search found that reduced CMTM8 expression was associated with a lower survival probability in lung squamous cell carcinoma progression (Figure 7).

\section{Conclusions and Prospects}

Lung cancer is one of the most common cancers, with a reported dramatic rise in mortality and morbidity. Research into the relationship of the potential tumor suppressor gene CMTM7 with lung cancer has so far focused on NSCLC, and identified a complex association that could exert either a positive or negative effect on tumor cells. CMTM research is still in its infancy, but investigations of clinical samples, patient prognostic data, in vitro and in vivo models, signal transduction pathways, and protein interactions provide a greater comprehension. Understanding the biological function and mechanism of CMTM in tumors will help provide a theoretical and experimental basis for effective tumor diagnosis, prognostic assessment, and improved sensitivity in radiotherapy, chemotherapy, and targeted therapy.

\section{Acknowledgments}

We thank the sponsorship from the Department of Technology of Liaoning Province (2017225010 to L.W). We thank Sarah Williams, PhD, from Liwen Bianji, Edanz Group China (www.liwenbianji.cn), for editing the English text of a draft of this manuscript.

\section{Author Contributions}

Writing: KW, HG, QY, and YL; Review and editing: XL and LW.

\section{Competing Interests}

The authors have declared that no competing interest exists.

\section{References}

1. Lu J, Wu QQ, Zhou YB, Zhang KH, Pang BX, Li L, et al. Cancer Research Advance in CKLF-like MARVEL Transmembrane Domain Containing Member Family (Review). Asian Pacific journal of cancer prevention : APJCP. 2016; 17: 2741-4.

2. Han W, Ding P, Xu M, Wang L, Rui M, Shi S, et al. Identification of eight genes encoding chemokine-like factor superfamily members 1-8 (CKLFSF1-8) by in silico cloning and experimental validation. Genomics. 2003; 81: 609-17.

3. Han W, Lou Y, Tang J, Zhang Y, Chen Y, Li Y, et al. Molecular cloning and characterization of chemokine-like factor 1 (CKLF1), a novel human cytokine with unique structure and potential chemotactic activity. The Biochemical journal. 2001; 357: 127-35.

4. Yang GY, Chen X, Sun YC, Ma CL, Qian G. Chemokine-like factor 1 (CLFK1) is over-expressed in patients with atopic dermatitis. International journal of biological sciences. 2013; 9: 759-65.

5. Zhong J, Wang Y, Qiu X, Mo X, Liu Y, Li T, et al. Characterization and expression profile of CMTM3/CKLFSF3. Journal of biochemistry and molecular biology. 2006; 39: 537-45

6. Xu M, Han W, Qian M, Ma X, Ding P, Wang Y, et al. Last intron of the chemokine-like factor gene contains a putative promoter for the downstream CKLF super family member 1 gene. Biochem Biophys Res Commun. 2004: 313: 135-41.

7. Shi S, Rui M, Han W, Wang Y, Qiu X, Ding P, et al. CKLFSF2 is highly expressed in testis and can be secreted into the seminiferous tubules. Int $J$ Biochem Cell Biol. 2005; 37: 1633-40

8. Gao DH, Hu H, Fang ZW, Huo F, Wang HR, Xu KX, et al. Research Advances in Chemokine-like Factor Super Family Member 8. Zhongguo Yi Xue Ke Xue Yuan Xue Bao. 2016; 38: 746-9.

9. Li $\mathrm{H}$, Guo $X$, Shao L, Plate M, Mo X, Wang $Y$, et al. CMTM5-v1, a four-transmembrane protein, presents a secreted form released via a vesicle-mediated secretory pathway. BMB reports. 2010; 43: 182-7.

10. Wang Y, Li J, Cui Y, Li T, Ng KM, Geng H, et al. CMTM3, located at the critical tumor suppressor locus 16q22.1, is silenced by CpG methylation in carcinomas and inhibits tumor cell growth through inducing apoptosis. Cancer research. 2009; 69: 5194-201.

11. Shao L, Cui Y, Li H, Liu Y, Zhao H, Wang Y, et al. CMTM5 exhibits tumor suppressor activities and is frequently silenced by methylation in carcinoma cell lines. Clin Cancer Res. 2007; 13: 5756-62.

12. Wang L, Wu C, Zheng Y, Qiu X, Fan H, Han W, et al. Molecular cloning and characterization of chemokine-like factor super family member 1 
(CKLFSF1), a novel human gene with at least 23 alternative splicing isoforms in testis tissue. Int J Biochem Cell Biol. 2004; 36: 1492-501.

13. Jin C, Wang Y, Han W, Zhang Y, He Q, Li D, et al. CMTM8 induces caspase-dependent and -independent apoptosis through a mitochondria-mediated pathway. J Cell Physiol. 2007; 211: 112-20.

14. Jin C, Ding P, Wang Y, Ma D. Regulation of EGF receptor signaling by the MARVEL domain-containing protein CKLFSF8. FEBS Lett. 2005; 579: 6375-82.

15. Zhang W, Mendoza MC, Pei X, Ilter D, Mahoney SJ, Zhang Y, et al. Down-regulation of CMTM8 induces epithelial-to-mesenchymal transition-like changes via c-MET/extracellular signal-regulated kinase (ERK) signaling. J Biol Chem. 2012; 287: 11850-8.

16. Zhang M, Xu Y, Liu Y, Cheng Y, Zhao P, Liu H, et al. Chemokine-Like Factor 1 (CKLF-1) is Overexpressed in Keloid Patients: A Potential Indicating Factor for Keloid-Predisposed Individuals. Medicine. 2016; 95 : e3082.

17. Chrifi I, Louzao-Martinez L, Brandt M, van Dijk CGM, Burgisser P, Zhu $\mathrm{C}$, et al. CMTM3 (CKLF-Like Marvel Transmembrane Domain 3) Mediates Angiogenesis by Regulating Cell Surface Availability of VE-Cadherin in Endothelial Adherens Junctions. Arteriosclerosis, thrombosis, and vascular biology. 2017; 37: 1098-114.

18. Li G, Li GY, Ji HJ, Zhao WJ, Chu SF, Chen NH. [Effect of testosterone on the expression of CMTM family of the male spermatogenesis suppression rats]. Yao xue xue bao $=$ Acta pharmaceutica Sinica. 2010; 45: 995-1000.

19. Li T, Han W, Yang T, Ding P, Rui M, Liu D, et al. Molecular cloning and identification of mouse Cklfsf2a and Cklfsf2b, two homologues of human CKLFSF2. Int J Biochem Cell Biol. 2006; 38: 420-9.

20. Zhang JW, Liu TF, Chen XH, Liang WY, Feng XR, Wang L, et al. Validation of aspirin response-related transcripts in patients with coronary artery disease and preliminary investigation on CMTM5 function. Gene. 2017; 624: 56-65.

21. Niu JH, Bao L, Zhang Y, Li JL, Li LD, Xie M, et al. [Abnormally lower expression of $\mathrm{cmtm} 5$ gene in bone marrow cells from patients with multiple myeloma]. Zhongguo shi yan xue ye xue za zhi. 2010; 18: 363-7.

22. Ji Y, Zhang H, Yuan H, Yang GP, Zhang K, Xie LH. [Expression of chemokine like factor-1 in nephridial tissue of lupus nephritis]. Zhong nan da xue xue bao Yi xue ban = Journal of Central South University Medical sciences. 2007; 32: 490-3.

23. Niu J, Li H, Zhang Y, Li J, Xie M, Li L, et al. Aberrant expression of CKLF-like MARVEL transmembrane member 5 (CMTM5) by promoter methylation in myeloid leukemia. Leukemia research. 2011; 35: 771-6.

24. Dunne PD, O'Reilly PG, Coleman HG, Gray RT, Longley DB, Johnston PG, et al. Stratified analysis reveals chemokine-like factor (CKLF) as a potential prognostic marker in the MSI-immune consensus molecular subtype CMS1 of colorectal cancer. Oncotarget. 2016; 7: 36632-44.

25. Bei C, Zhang Y, Wei R, Zhu X, Wang Z, Zeng W, et al. Clinical significance of CMTM4 expression in hepatocellular carcinoma. OncoTargets and therapy. 2017; 10: 5439-43.

26. Mezzadra R, Sun C, Jae LT, Gomez-Eerland R, de Vries E, Wu W, et al. Identification of CMTM6 and CMTM4 as PD-L1 protein regulators. Nature. 2017; 549: 106-10.

27. Zhang H, Zhang X, Yuan X, Wang L, Xiao Y. MicroRNA-205 inhibits renal cells apoptosis via targeting CMTM4. Iran J Basic Med Sci. 2015; 18: 1020-6.

28. Shen Z, Chen X, Li Q, Zhou C, Xu Y, Yu R, et al. Elevated methylation of CMTM3 promoter in the male laryngeal squamous cell carcinoma patients. Clinical biochemistry. 2016; 49: 1278-82.

29. Yuan W, Li T, Mo X, Wang X, Liu B, Wang W, et al. Knockdown of CMTM3 promotes metastasis of gastric cancer via the STAT3/Twist1/EMT signaling pathway. Oncotarget. 2016; 7: 29507-19.

30. Han T, Shu T, Dong S, Li P, Li W, Liu D, et al. Chemokine-like factor-like MARVEL transmembrane domain-containing 3 expression is associated with a favorable prognosis in esophageal squamous cell carcinoma. Oncol Lett. 2017; 13: 2982-8.

31. Zhang H, Zhang J, Nan X, Li X, Qu J, Hong Y, et al. CMTM3 inhibits cell growth and migration and predicts favorable survival in oral squamous cell carcinoma. Tumour Biol. 2015; 36: 7849-58.

32. Yuan W, Liu B, Wang $X$, Li T, Xue H, Mo X, et al. CMTM3 decreases EGFR expression and EGF-mediated tumorigenicity by promoting Rab5 activity in gastric cancer. Cancer Lett. 2017; 386: 77-86.

33. Su Y, Lin Y, Zhang L, Liu B, Yuan W, Mo X, et al. CMTM3 inhibits cell migration and invasion and correlates with favorable prognosis in gastric cancer. Cancer Sci. 2014; 105: 26-34.

34. Xu G, Dang C. CMTM5 is downregulated and suppresses tumour growth in hepatocellular carcinoma through regulating PI3K-AKT signalling. Cancer cell international. 2017; 17: 113.

35. Li W, Zhang S. CKLF-Like MARVEL Transmembrane Domain-Containing Member 3 (CMTM3) Inhibits the Proliferation and
Tumorigenisis in Hepatocellular Carcinoma Cells. Oncol Res. 2017; 25 : 285-93.

36. Gao D, Hu H, Wang Y, Yu W, Zhou J, Wang X, et al. CMTM8 inhibits the carcinogenesis and progression of bladder cancer. Oncology reports. 2015; 34: 2853-63.

37. Zhang S, Pei X, Hu H, Zhang W, Mo X, Song Q, et al. Functional characterization of the tumor suppressor CMTM8 and its association with prognosis in bladder cancer. Tumour Biol. 2016; 37: 6217-25.

38. Hu F, Yuan W, Wang X, Sheng Z, Yuan Y, Qin C, et al. CMTM3 is reduced in prostate cancer and inhibits migration, invasion and growth of LNCaP cells. Clin Transl Oncol. 2015; 17: 632-9.

39. Xie J, Yuan Y, Liu Z, Xiao Y, Zhang X, Qin C, et al. CMTM3 is frequently reduced in clear cell renal cell carcinoma and exhibits tumor suppressor activities. Clin Transl Oncol. 2014; 16: 402-9.

40. Li Z, Xie J, Wu J, Li W, Nie L, Sun X, et al. CMTM3 inhibits human testicular cancer cell growth through inducing cell-cycle arrest and apoptosis. PLoS One. 2014; 9: e88965.

41. Wang J, Zhang G, Zhang Y, Luo Y, Song Q, Qiu X, et al. CMTM1_v17 is a novel potential therapeutic target in breast cancer. Oncology reports. 2014; 32: 1829-36

42. Delic S, Thuy A, Schulze M, Proescholdt MA, Dietrich P, Bosserhoff AK, et al. Systematic investigation of CMTM family genes suggests relevance to glioblastoma pathogenesis and CMTM1 and CMTM3 as priority targets. Genes Chromosomes Cancer. 2015; 54: 433-43.

43. Liu Q, Su Y, Jiang GC, Zhou ZL, Liu BC, Bu L, et al. Change of CMTM7 expression, a potential tumor suppressor, is associated with poor clinical outcome in human non-small cell lung cancer. Chin Med J (Engl). 2013; 126: 3006-12.

44. Zhang W, Qi H, Mo X, Sun Q, Li T, Song Q, et al. CMTM8 is Frequently Downregulated in Multiple Solid Tumors. Applied immunohistochemistry \& molecular morphology : AIMM. 2017; 25: $122-8$.

45. Cai B, Xiao Y, Li Y, Zheng S. CMTM5 inhibits renal cancer cell growth through inducing cell-cycle arrest and apoptosis. Oncol Lett. 2017; 14: 1536-42.

46. Li T, Cheng Y, Wang P, Wang W, Hu F, Mo X, et al. CMTM4 is frequently downregulated and functions as a tumour suppressor in clear cell renal cell carcinoma. J Exp Clin Cancer Res. 2015; 34: 122.

47. Wang L, Wu C, Zheng Y, Qiu X, Wang L, Fan H, et al. Molecular cloning and characterization of chemokine-like factor super family member 1 (CKLFSF1), a novel human gene with at least 23 alternative splicing isoforms in testis tissue. Int J Biochem Cell Biol. 2004; 36: 1492-501.

48. Zhu BM, Li T, Zhou YL, Song QS, Wang L. [CMTM1-v17, a new potential corepressor of androgen receptor]. Beijing da xue xue bao Yi xue ban = Journal of Peking University Health sciences. 2007; 39: 388-93.

49. Si J, Zhang P, Tian D, Wang X, Ma Y, Zhang J, et al. CMTM1_v17 is associated with chemotherapy resistance and poor prognosis in non-small cell lung cancer. World journal of surgical oncology. 2017; 15: 34

50. Yuan YQ, Xiao YB, Liu ZH, Zhang XW, Xu T, Wang XF. [Research advances in CKLF-like MARVEL transmembrane domain containing member 5]. Zhongguo Yi Xue Ke Xue Yuan Xue Bao. 2012; 34: 625-8.

51. Yuan YQ, Zhang YX, Liu ZH, Qin CP, Sheng ZZ, Xu T, et al. [Expression and significance of CMTM5 and epidermal growth factor receptor in prostate cancer]. Beijing da xue xue bao Yi xue ban = Journal of Peking University Health sciences. 2015; 47: 571-6.

52. Walsh CM, Rose DN, Dubrowski A, Ling SC, Grierson LE, Backstein D, et al. Learning in the simulated setting: a comparison of expert-, peer-, and computer-assisted learning. Acad Med. 2011; 86: S12-6.

53. Zhang H, Nan X, Li X, Chen Y, Zhang J, Sun L, et al. CMTM5 exhibits tumor suppressor activity through promoter methylation in oral squamous cell carcinoma. Biochem Biophys Res Commun. 2014; 447: 304-10

54. Xiao Y, Yuan Y, Zhang Y, Li J, Liu Z, Zhang X, et al. CMTM5 is reduced in prostate cancer and inhibits cancer cell growth in vitro and in vivo. Clin Transl Oncol. 2015; 17: 431-7.

55. Xiao YB, Xie J, Zhang GX, Li J, Hao YC, Zhang XW, et al. [Inhibitory effect of CMTM5 on xenografted human prostatic cancer in nude mice]. Zhonghua Nan Ke Xue. 2012; 18: 195-9.

56. Xu T, Li J, Xiao YB, Liu ZH, Li Q, Wang XF. [CMTM5 inhibits the tumor cell behavior of prostate cancer by downregulation of HER2]. Beijing Da Xue Xue Bao Yi Xue Ban. 2010; 42: 386-90.

57. Zhu X, Qi G, Li C, Bei C, Tan C, Zhang Y, et al. Expression and Clinical Significance of CMTM6 in Hepatocellular Carcinoma. DNA Cell Biol. 2019; 38: 193-7.

58. Guan X, Zhang C, Zhao J, Sun G, Song Q, Jia W. CMTM6 overexpression is associated with molecular and clinical characteristics of malignancy and predicts poor prognosis in gliomas. EBioMedicine. 2018; 35: 233-43. 
59. Burr ML, Sparbier CE, Chan YC, Williamson JC, Woods K, Beavis PA, et al. CMTM6 maintains the expression of PD-L1 and regulates anti-tumour immunity. Nature. 2017; 549: 101-5.

60. Han W, Lou Y, Tang J, Zhang Y, Chen Y, Li Y, et al. Molecular cloning and characterization of chemokine-like factor 1 (CKLF1), a novel human cytokine with unique structure and potential chemotactic activity. The Biochemical journal. 2001; 357: 127-35.

61. Li H, Li J, Su Y, Fan Y, Guo X, Li L, et al. A novel 3p22.3 gene CMTM7 represses oncogenic EGFR signaling and inhibits cancer cell growth. Oncogene. 2014; 33: 3109-18.

62. Liu B, Su Y, Li T, Yuan W, Mo X, Li H, et al. CMTM7 knockdown increases tumorigenicity of human non-small cell lung cancer cells and EGFR-AKT signaling by reducing Rab5 activation. Oncotarget. 2015; 6: 41092-107.

63. Liu Q, Yu SU, Pei GT, Wang S, Yang YS, Min XJ, et al. [Effects of RNA Interference CMTM7 on Apoptosis of Lung Adenocarcinoma A549 Cells]. Progress in Modern Biomedicine. 2016; 16: 3431-4.

64. Chen SQ, Du Y, Huang YM, Wang X, Gu QL, Dong ZM. [Effects of novel human chemokine-like factor superfamily 8 on proliferation and EGFR expression of HL-60 cells]. Zhongguo shi yan xue ye xue za zhi. 2007; 15: 458-61.

65. Hu H, Chen JW, Xu KX, Wang D, Wang Y, Wang GW, et al. [Expressions of CMTM8 and E-cadherin in primary and metastatic clear cell renal cell carcinoma]. Beijing da xue xue bao Yi xue ban = Journal of Peking University Health sciences. 2013; 45: 537-41. 